\title{
Planificación Estocástica de la Producción para Aceite de Oliva Virgen
}

\author{
P. Cano Marchal, D. Martínez Gila, J. Gámez García, J. Gómez Ortega \\ Grupo de Robótica, Automática y Visión por Computador \\ Universidad de Jaén. Campus de Excelencia Internacional Agroalimentario (ceiA3). \\ \{pcano,jggarcia,juango\}@ujaen.es
}

Optimización, Sistema de apoyo a la decisión, Aceite de oliva

\section{Resumen}

La calidad y cantidad de aceite de oliva virgen están influenciadas por el efecto de las variables tecnológicas durante el proceso de producción en base a las propiedades de las aceitunas de entrada. Dado que la calidad de las aceitunas evoluciona durante la campaña de recolección, una cuestión relevante es la determinación sistemática del momento de recolección para maximizar el beneficio total de campaña. Este trabajo propone un método para determinar el plan óptimo de producción para la campaña considerando la naturaleza estocástica del problema, presentando los resultados de su aplicación a diferentes escenarios de producción.

\section{Introducción}

El retorno económico de la producción de aceite de oliva virgen (AOV) depende del grado de extracción de aceite de la aceituna - normalmente referido en términos de rendimiento industrial o agotamiento-, la calidad del AOV y los costos incurridos durante la producción. Desafortunadamente, la obtención de buenos agotamientos y alta calidad son objetivos contrapuestos, lo que otorga una importancia fundamental a esta consideración en la toma de decisiones en el proceso. [9, 3].

La evolución de las propiedades del fruto en campo, el método de recolección y los valores de las variables tecnológicas durante el proceso de extracción influyen en el valor final de cada uno de estos objetivos [1]. Por tanto, antes de procesar un lote de aceituna para producir AOV se debe decidir qué punto de la superficie de compromiso entre agotamiento, calidad y costo tomar como objetivo. Esta decisión define un objetivo de producción que implica la elección de un cierto conjunto de variables de proceso para conseguir alcanzar dicho objetivo.

La influencia de las variables de proceso en la calidad y agotamiento, así como su medición en línea, han sido temas de intensa actividad investigadora [7, 8] y [15], respectivamente. El esfuerzo dedicado a estos temas está justificado por la gran importancia del tema, dado que es precisamente la interacción entre las distintas variables de proceso lo que finalmente determina la salida del proceso, es decir, los valores de calidad y agotamiento obtenidos.

Apoyados en esta investigación, una cuestión relevante es sugerir sistemáticamente cuál sería el mejor objetivo de elaboración para un cierto lote de aceitunas dado, asumiendo que el objetivo es maximizar el rendimiento económico de la actividad. En [5] y [4] se ha considerado este problema en el contexto de un sistema general para la definición y actualización de referencias adecuadas para las variables de proceso de elaboración de aceite de oliva (PEAOV). Sin embargo, asumir que el lote de aceitunas a procesar está ya en la fábrica implica que las propiedades de éstas ya están fijadas. Dado que las propiedades de la aceituna evolucionan a lo largo de la campaña y que son un factor fundamental en el proceso [11, 14], es muy relevante considerar qué valores deben tener estas propiedades para maximizar el beneficio para toda la campaña.

El objetivo de este trabajo es profundizar en el trabajo preliminar incluido en [6] y proponer un método para determinar un plan de producción óptimo para toda la campaña, i.e., definir la cantidad de cada calidad de AOV que maximiza el beneficio y en qué momento debe ser producido, considerando las restricciones pertinentes y la naturaleza estocástica del problema.

El resto del trabajo se organiza como sigue: la Sección 2 aborda la parte teórica, presentando la función objetivo, restricciones y dos enfoques para obtener los valores de los parámetros del modelo. La Sección 3 muestra los resultados obtenidos usando el método propuesto para diferentes escenarios, con la Sección 4 finalizando el trabajo con la presentación de la conclusiones y futuras líneas de trabajo.

\section{Definición del Problema de Optimización}

Las siguientes Secciones presentan en detalle la definición del problema de optimizació y la conveniencia e implicaciones de considerar la naturaleza estocástica del problema. 


\subsection{Planteamiento general}

La producción total de AOV llevada a cabo a lo largo de la campaña se puede considerar como una colección de lotes de AOV producidos en distintos períodos de tiempo, donde cada lote está caracterizado por la propiedades del fruto $(\nu)$, los valores de las variables implicadas en la transformación de aceituna en aceite desde el campo hasta la bodega $(\xi)$ y la calidad del AOV obtenido $(\phi)$.

Un aspecto interesante a considerar es que dos AOV de la misma calidad pueden proporcionar beneficios diferentes, dependiendo de la forma de comercialización de los mismos, siendo un ejemplo claro la diferencia entre la venta de AOV a granel y envasado. Sin embargo, típicamente existen restricciones en la capacidad de venta de envasado de una entidad, mientras que el mercado de granel se puede considerar capaz de absorber toda la producción. Estas consideraciones sugieren la conveniencia de introducir el concepto de producto en la formalización del problema de optimización. Un producto está caracterizado por un umbral de calidad y un método de comercialización asociado.

El plan de producción, por tanto, requiere definir la cantidad de cada producto a producir durante toda la campaña. Si empleamos el índice $i=1,2, \ldots, I$ para enumerar los distintos períodos de producción y $k=1,2, \ldots K$ para referir los productos, el plan de producción se puede formalizar como una matriz $P \in \mathbb{R}^{I \times K}$ :

$$
P=\left[\begin{array}{cccccc}
a_{11} & a_{12} & \ldots & a_{i 1} & \ldots & a_{1 K} \\
a_{21} & a_{22} & \ldots & a_{i 2} & \ldots & a_{2 K} \\
\vdots & \vdots & \ldots & \vdots & \ldots & \vdots \\
a_{i 1} & a_{i 2} & \ldots & a_{i k} & \ldots & a_{i K} \\
\vdots & \vdots & \ldots & \vdots & \ldots & \vdots \\
a_{I 1} & a_{I 2} & \ldots & a_{I k} & \ldots & a_{I K}
\end{array}\right]
$$

Aquí, cada entrada $a_{i k}$ de la matriz representa la cantidad de aceitunas que se dedican a la producción del producto $k$ en el período $i$, cada columna describe el plan de producción para un producto específico y cada fila representa la distribución de la producción para un instante de tiempo determinado. Las siguientes Secciones detallan los diferentes aspectos considerados en la construcción del problema de optimización.

\subsubsection{Análisis de un período de producción}

Comenzamos el análisis considerando un cierto período de producción $i$. En este período las propiedades del fruto $\nu_{i}$ se pueden considerar fijas, dado que son las disponibles para dicho período.

El beneficio obtenido al procesar un lote específico de un cierto producto y vender el AOV es simplemente el producto de la cantidad de AOV y el precio de venta menos los costos de producción. Matemáticamente:

$$
J_{i k}^{b}=s_{i k} \pi_{k}-a_{i k} \kappa_{i k},
$$

donde $s_{i k}$ representa la cantidad total de producto $k$ producido, $\pi_{k}$ el precio de venta del producto, $\kappa_{i k}$ el coste total de operación por unidad de aceituna procesada y $a_{i k}$ la cantidad de aceitunas procesadas en el lote dedicadas al producto $k$.

La cantidad de aceite obtenido $\left(s_{i k}\right)$ se puede expresar como la cantidad de aceitunas procesadas multiplicadas por el rendimiento industrial de extracción $\left(\rho_{i k}\right)$. Por su parte, $\pi_{k}$ depende de la calidad del producto, mientras que $\kappa_{i k}$ lo hace de la forma en que las aceitunas fueron procesadas, es decir, de los valores de las variables de proceso $\left(\xi_{i k}\right)$. Incluyendo estas consideraciones, la Ec. (2) se puede expresar como:

$$
J_{i k}^{b}=a_{i k}\left(\rho_{i k} \pi_{k}\left(\phi_{k}\right)-\kappa_{i k}\left(\xi_{i k}\right)\right) .
$$

Es relevante remarcar que $\phi_{k}$ es una propiedad del producto y que es fija durante toda la campaña, por lo que no tiene índice $i$. Por simplicidad, el precio de venta $\pi_{k}$ también se considera constante durante la campaña y que depende únicamente en el producto en cuestión. Como se ha comentado anteriormente, $\rho_{i k}$ es una función de las propiedades del fruto $\left(\nu_{i}\right)$ y las variables de proceso $\left(\xi_{i k}\right)$. Si incluimos explícitamente esta dependencia en Eq. (3) tenemos:

$$
J_{i k}^{b}=a_{i k}\left(\rho_{i k}\left(\xi_{i k}, \nu_{i}, \phi_{k}\right) \pi_{k}\left(\phi_{k}\right)-\kappa_{i k}\left(\xi_{i k}\right)\right) .
$$

Esta ecuación establece que el beneficio para un cierto lote de aceitunas es una función de las propiedades de dichas aceitunas $\left(\nu_{i}\right)$, la forma en que se transforman en $\mathrm{AOV}\left(\xi_{i k}\right)$ y la calidad del producto $\left(\phi_{k}\right)$, i.e., $J_{i k}^{b}=J^{b}\left(a_{i k}, \nu_{i}, \xi_{i k}, \phi_{k}\right)$.

Sin embargo, $\phi_{k}$ es un valor fijo, y la conocida relación de compromiso entre calidad y agotamiento implica que fijar un cierto valor de $\phi_{k}$ restringe la libertad de elección de $\xi_{i k}$, influyendo, por tanto, en el valor de $\rho_{i k}$. Es más, $\nu_{i}$ también es fijo, dado que es valor disponible en el período en cuestión. Para explicitar que la única variable realmente elegible es $\xi_{i k}$, podemos utilizar la notación $J_{i k}^{b}=J_{i k}^{b}\left(\xi_{i k} \mid \nu_{i}, \phi_{k}\right)$. Por conveniencia, definimos:

$$
\gamma_{i k}\left(\xi_{i k} \mid \nu_{i}, \phi_{k}\right)=\rho_{i k}\left(\xi_{i k} \mid \nu_{i}, \phi_{k}\right) \pi_{k}\left(\phi_{k}\right)-\kappa_{i k}\left(\xi_{i k}\right) .
$$

Esta cantidad $\gamma_{i k}$ tiene la interpretación directa de ser el beneficio por unidad de aceituna procesada para el producto $k$, y permite expresar Eq. (4) de manera compacta como:

$$
J_{i k}^{b}\left(a_{i k}, \xi_{i k} \mid \nu_{i}, \phi_{k}\right)=a_{i k} \gamma_{i k}\left(\xi_{i k} \mid \nu_{i}, \phi_{k}\right) .
$$

La inspección de la Ecuación anterior muestra que el beneficio obtenido para un lote de un cierto producto se puede 
factorizar en dos términos: la cantidad de aceitunas procesadas $\left(a_{i k}\right)$ y un factor que depende del tipo de producto $\left(\pi_{k}\right)$, las propiedades de las aceitunas $\left(\nu_{i}\right)$ y la manera en que se procesan $\left(\xi_{i k}\right)$, pero no depende explícitamente de la cantidad de aceitunas procesada. Puesto que ambos términos, por su definición, son independientes es claro que el óptimo de Eq. (6) se puede obtener optimizando de forma independiente cada término: $a_{i k}$ and $\gamma_{i k}$.

La optimización del segundo término es el problema de encontrar el valor de $\xi_{i k}$ que maximice $\rho_{i k}$ teniendo en consideración el precio de venta y los costes de producción, sujeto a las propiedades de las aceitunas disponibles $\nu_{i}$ y el requerimiento de obtener un AOV de calidad $\phi_{k}$. Matemáticamente:

$$
\underset{\xi_{i k}}{\operatorname{maximize}} \gamma_{i k}=\rho_{i k}\left(\xi_{i k} \mid \nu_{i}, \phi_{k}\right) \pi_{k}\left(\phi_{k}\right)-\kappa_{i k}\left(\xi_{i k}\right) .
$$

Este problema se puede abordar utilizando las técnicas propuestas en [5] y [4], y se estudiará en más detalle en la Sección ??. Sin embargo, la observación clave es que se puede resolver de manera independiente al problema de planificación de la producción general, y su solución $\gamma_{i k}^{*}$ se puede considerar un parámetro fijo en el problema general.

Otro comentario importante es que el requisito de alcanzar un cierto valor de $\phi_{k}$ puede hacer que el problema de optimización no sea factible. En este caso se debe asegurar que no se asigna producción a dicho producto en ese período de tiempo, bien incluyendo una restricción explícita $a_{i k} \leq 0$, bien simplemente fijando $\gamma_{i k}^{*}=0$.

Una vez que $\gamma_{i k}^{*}$ está disponible se debe determinar el valor de $a_{i k}$. Si consideramos que únicamente se puede producir el producto $k$, entonces la solución obvia es que $a_{i k}$ debe ser tan grande como sea posible. Si, por otra parte, se pueden producir varios productos se debe elegir cuál de ellos proporciona el mayor retorno económico, sujeto a la cantidad total de aceitunas disponibles en el periodo:

$$
\begin{array}{ll}
\underset{a_{i k}}{\operatorname{maximize}} & \sum_{k} a_{i k} \gamma_{i k}^{*} \\
\text { subject to } & \sum_{k} a_{i k} \leq \bar{a}_{i} .
\end{array}
$$

La solución de este problema es claramente asignar la producción a aquellos productos cuyo valor de $\gamma_{i k}^{*}$ es mayor. Sin embargo, no es verdad en general de que la solución del problema de optimización global requiera asignar producción a cada instante de tiempo, por lo que no se puede optimizar cada instante de manera aislada. La siguiente Sección trata de la formulación del problema completo.

\subsubsection{Problema general básico}

De acuerdo con la discusión de la Sección anterior, se puede suponer que $\gamma_{i k}^{*}$ están disponibles para cada producto y período de producción, puesto que su valor es indepen- diente del plan de producción. De esta forma, el problema es una extensión de (7) y su función objetivo se puede formalizar como:

$$
\underset{a_{i k}}{\operatorname{maximizar}} \quad J=\sum_{i} \sum_{k} a_{i k} \gamma_{i k}^{*}
$$

Para definir correctamente el problema de optimización se deben introducir una serie de restricciones. Primero, el número total de aceitunas para la campaña está claramente acotado, por lo que una restricción en este sentido debe ser incluida. Por otro lado, las aceitunas van perdiendo humedad con el avance de la campaña, lo que disminuye su peso. Este efecto se debe tener en cuenta introduciendo un factor $\lambda_{i}$ inversamente proporcional al contenido de humedad del fruto en cada instante de tiempo:

$$
\sum_{i} \sum_{k} \lambda_{i} a_{i k} \leq \bar{a}
$$

Dos aspectos limitan la cantidad de fruto que se puede procesar en cada etapa: la capacidad de producción instalada en fábrica y la disponibilidad de fruto. Típicamente, la capacidad de producción de la fábrica se puede considerar como fija para la campaña, sin embargo, la disponibilidad de fruto sí que varía a lo largo del año. Estas consideraciones se pueden modelar con la restricción:

$$
\sum_{k} a_{i k} \leq \bar{a}_{i}
$$

Limitaciones sobre la disponibilidad de aceitunas adecuadas para la obtención de un nivel específico de calidad también se pueden incluir fácilmente de manera análoga a lo comentado en la Sección anterior. La cota de estas restricciones se puede calcular previamente teniendo en cuenta la cantidad de aceituna de las distintas calidad disponible en cada instante. Estas restricciones son:

$$
a_{i k} \leq \bar{a}_{i k} .
$$

Finalmente, la previamente comentada restricción sobre la capacidad de venta de la compañía se puede modelar con la restricción:

$$
\sum_{i} \rho_{i k} a_{i k} \leq \bar{a}_{k},
$$

donde se ha tenido en cuenta la transformación de aceitunas en aceite empleando el factor $\rho_{i k}$.

Las ecuaciones (8-12) configuran un modelo de optimización lineal sencillo que constituye la base del problema que nos ocupa.

\subsubsection{Inclusión de costes fijos}

Las soluciones del problema de optimización anterior tienden a asignar la producción de AOV lampante a los 
períodos más retrasados posibles. Esto se debe al efecto comentado de pérdida de humedad del fruto, lo que produce mayor rentabilidad debido al menor costo por la reducción de la cantidad de aceituna procesada para obtener una misma cantidad de AOV.

Sin embargo, la apertura de la instalación conlleva incurrir en costos, puesto que se debe contratar personal y suministro eléctrico, y parte de estos costos son fijos, puesto que no dependen de si efectivamente existe producción asignada al periodo o no. Existe, por tanto, un término de costos fijos asociado a la apertura de la instalación, independiente de si existe producción asignada o no.

Para modelar este efecto se deben introducir varias variables. Primero, una variable entera $\delta_{i}$ codifica si existe producción asignada a un cierto periodo. Las restricciones asociadas a estas variables utilizan la formulación big- $M$ [16]:

$$
\sum_{k} a_{i k} \leq M \delta_{i}
$$

Sin embargo, la inclusión de estas variables y restricciones no es suficiente para codificar el período de tiempo desde que se inicia la producción hasta que finaliza. Para lograr esto, se introducen dos nuevos conjuntos de variables binarias $\left(\epsilon_{i}^{A}\right.$ and $\left.\epsilon_{i}^{B}\right)$ junto con las restricciones:

$$
\begin{aligned}
\sum_{k, j \geq i} a_{j k} & \leq M \epsilon_{i}^{A} \\
\sum_{k, j \leq i} a_{j k} & \leq M \epsilon_{i}^{B} .
\end{aligned}
$$

Estas restricciones fuerzan $\epsilon_{i}^{A}$ a ser uno siempre que exista producción durante y posteriormente a dicho período, mientras que $\epsilon_{i}^{B}$ es uno si existe producción en dicho período o antes de $i$.

Para cada instante de tiempo existen cuatro posibilidades:

- La producción aún no ha comenzado. En este escenario $\epsilon^{A}$ vale 1 , mientras que $\epsilon^{B}$ vale 0 .

- Existe producción asignada al período. Aquí, tanto $\epsilon^{A}$ como $\epsilon^{B}$ valen 1 .

- La producción ha comenzado ya y aún no ha terminado, pero no hay producción asignada al período considerado. En este caso, de nuevo tanto $\epsilon^{A}$ como $\epsilon^{B}$ valen 1.

- La producción ya ha finalizado y, lógicamente, no existe producción asignada al periodo. En este escenario, $\epsilon^{A}$ vale 0 , mientras que $\epsilon^{B}$ vale 1 .

Por tanto, el valor absoluto de la diferencia entre $\epsilon^{A}$ y $\epsilon^{B}$ es 1 cuando la fábrica bien no ha comenzado la producción, bien ya la ha terminado, y 0 cuando existe producción asignada al período o justo antes y después del mismo. Por tanto, la cantidad de instantes considerados menos la suma de los valores absolutos de las diferencias entre $\epsilon^{A}$ y $\epsilon^{B}$ proporcionan el número de instantes en que la fábrica está abierta.

Esta discusión se puede codificar utilizando dos nuevos conjuntos de variables binarias $\left(\xi^{A}\right.$ and $\left.\xi^{B}\right)$ asociadas a las siguientes restricciones:

$$
\begin{aligned}
& \xi_{i}^{A}-\xi_{i}^{B}=\epsilon_{i}^{A}-\epsilon_{i}^{B}, \\
& \xi_{i}^{A}+\xi_{i}^{B} \leq 1 .
\end{aligned}
$$

De esta manera, el número total de períodos abiertos viene dado por $t=n-\sum_{i}\left(\xi_{i}^{A}+\xi_{i}^{B}\right)$, y la función de coste se puede aumentar con un término que penalice esta variable:

$$
\underset{a_{i k}}{\operatorname{maximize}} \quad J=\sum_{i} \sum_{k} a_{i k} \gamma_{i k}^{*}-C t
$$

Desafortunadamente, la inclusión de estas restricciones supone perder la naturaleza lineal del problema de optimización, pasando a ser un problema de programación lineal entera mixta.

\subsection{Incertidumbre en los parámetros del problema}

La planificación de la producción de AOV para toda la campaña se ve influenciada por distintas fuentes de incertidumbre que deben ser tenidas en cuenta a la hora de definir el problema de optimización, puesto que tiene influencia tanto en los valores de los parámetros como en la propia estructura del problema.

La evolución de las propiedades del fruto claramente constituye una variable aleatoria cuyo valor esperado se puede inferir de la literatura [11] [14] y datos históricos de cada compañía, pero cuyo valor preciso para cada campaña es desconocido. Esta incertidumbre supone un elemento fundamental, puesto que la idea general del método es elegir cuándo cosechar tomando como base estas propiedades del fruto.

Para tener en cuenta esta incertidumbre se pueden considerar el estudio de la solución asignada a diversos escenarios. Sin embargo, simplemente considerar estos escenarios y resolverlos de manera aislada es equivalente a suponer que se conocen las propiedades futuras de las propiedades en el momento de la planificación, lo cual, obviamente, no es cierto. Una alternativa es resolver el problema considerando el valor esperado de los parámetros sujetos a incertidumbre, lo que proporcional la solución de valor esperado del problema. Finalmente, para tener en cuenta que las propiedades del fruto se van descubriendo conforme avanza la campaña, se puede definir un problema de 

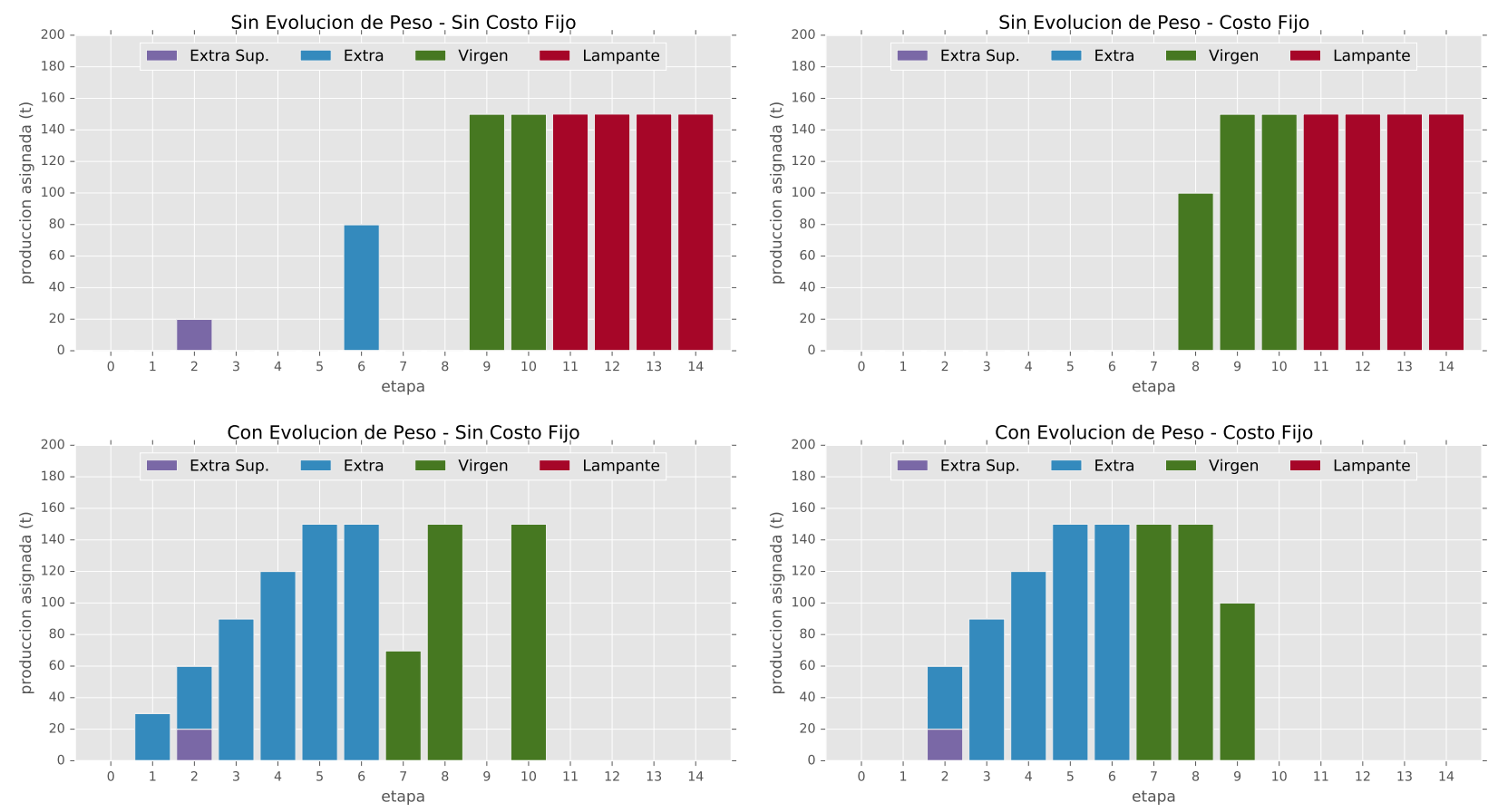

Figura 1: Plan de producción para evolución de las propiedades del fruto y precios fijos para cuatro escenarios. Los gráficos de la izquierda (derecha) no (sí) incluyen costos fijos. Los gráficos de la fila superior (inferior) no (sí) incluyen la evolución de la humedad.

optimización estocástica multietapa que incluya las correspondientes restricciones de no anticipatividad [2]. De esta manera, cada etapa de producción se planifica de acuerdo con la información de la que es realista suponer que se dispone: la evolución del fruto hasta el momento, y las expectativas de evolución futura. No es trivial, en general, determinar si el beneficio adicional de considerar el problema multietapa compensa la complejidad adicional respecto a la solución de valor esperado. La Sección 3 muestra algunos resultados referentes a esta discusión.

Los precios de los productos conforman otro conjunto de parámetros importante que puede ser considerado como aleatorio. Cambios relativos entre los distintos productos alteran la rentabilidad de cada uno de ellos, lo que provoca un potencial impacto en la solución del problema. En este caso no es trivial modelar el momento en que la información de los precios es revelada y si existe espacio para tomar decisiones basadas en esta información. Si nueva información se descubre a medida que se desarrolla la campaña estaríamos ante un escenario de optimización multietapa similar al obtenido al considerar la evolución del fruto. Si, por otro lado, consideramos que el valor de los precios se revela cuando la producción ya ha sido completada no estaríamos ante un escenario multietapa.

\section{Resultados y Discusión}

Esta Sección presenta las soluciones obtenidas empleando el método propuesto a un conjunto e escenarios. Los problemas de optimización se implementaron utilizando Pyomo [12] y PySP [18] mediante la definición de un modelo base determinista y varios árboles de escenario que definen las etapas del problema y los valores de los parámetros en cada escenario. La solución de los problemas se llevó a cabo utilizando PySP and Gurobi [10].

Todos los escenarios consideran un incremento progresivo de la capacidad de recolección $\left(\bar{a}_{i}\right)$ durante las primeras semanas, modelando la incorporación gradual de olivareros a la tareas de cosecha. Datos históricos de la evolución de grasa y humedad fueron proporcionados por un laboratorio experto en el sector y los parámetros relacionados con los modelos de producción se obtuvieron empleando los modelos presentados en [4].

\subsection{Inclusión de costos fijos y decremento de humedad}

El primer análisis consiste en la comparación de los planes de producción obtenidos considerando una evolución determinista de las propiedades de la aceituna y precios cuando se incluyen costos fijos y la evolución de la humedad en el modelo. La Figura 1 muestra los resultados obtenidos. El gráfico superior izquierdo muestra el resulta- 
Cuadro 1: Escenarios considerados en la Sección 3.2.

\begin{tabular}{llll} 
& Sin Daño & $\begin{array}{l}\text { Daño en el } \\
\text { instante 1 }\end{array}$ & $\begin{array}{l}\text { Daño en el } \\
\text { instante 3 }\end{array}$ \\
\hline $\begin{array}{l}2015 \\
\text { cios } \\
2016 \quad \text { Pre- } \\
\text { cios } \\
\text { probabilidad }\end{array}$ & A-D0 & A-D1 & A-D3 \\
\hline
\end{tabular}

do cuando no se consideran ninguno de estos dos efectos en el modelo. Como se puede ver, la producción se asigna exclusivamente basada en los valores más altos de $\rho_{i k}^{*}$ que se concentran al final de la campaña y asignando producción esporádica a AOV de alta calidad. El gráfico superior derecho incluye la consideración de los costos fijos, por lo que minimiza el número de días dedicado a la producción. Con el nivel de precios considerado en el escenario (escenario I del Cuadro ??), la producción se asigna completamente al final de campaña, no produciéndose AOV de alta calidad. Cuando se tiene en cuenta el decremento de peso de las aceitunas en campo debido a la pérdida de humedad (gráfico inferior izquierdo), se comprueba que la producción se traslada hacia el inicio de la campaña. Finalmente, la inclusión de costes fijos en el gráfico inferior derecho de nuevo reduce el número de días dedicados a la producción, frente a la solución proporcionada sin incluirlos.

\subsection{Precios y Evolución de Fruto Estocástica}

Esta Sección analiza seis escenarios diferentes: dos niveles de precios, correspondientes a los años 2015 y 2016 y a los cuales se le asigna la misma probabilidad, y tres casos de evolución de las propiedades del fruto: evolución sin incidencias, incidencia en el primer período de producción y en el tercero, respectivamente. La probabilidad de cada uno de estos escenarios está definida por el parámetro $p$ de acuerdo con el Cuadro 1 .

La evolución de la calidad de las aceitunas se revela según avanza la estación, por los que se pueden tomar decisiones basadas en los valores efectivamente tomados por las variables hasta el momento. L Figura 2 muestra los diferentes planes de producción obtenidos en la Sección. Las columnas corresponden a los tres escenarios de evolución de la calidad. La primera y la segunda fila muestran el plan de producción óptimo suponiendo que existe información perfecta para los niveles de precios A y B respectivamente. Como se muestra en la Figura, los planes coinciden para los escenarios D1 y D3, siendo distintos en el caso en que no se producen daños en el fruto. La tercera fila presenta la solución al problema multietapa, mientras que las filas 4 a 6 muestran las solución del problema de valor esperado para $p=0.1, p=0.3$ y $p=0.7$, respectivamente.

Es importante hacer notar que la solución de valor espe-
Cuadro 2: Valor de la Información Perfecta (VPI) and Valor de la solución estocástica (VSS) para los diferentes valores de $p$ considerados en la Sección 3.2.

\begin{tabular}{ccccc} 
& $p=0.1$ & $p=0.3$ & $p=0.5$ & $p=0.7$ \\
\hline VPI\% & 0.32 & 0.27 & 0.12 & 0.04 \\
VSS\% & 0.34 & 0.32 & 0.31 & 0.20 \\
\hline
\end{tabular}

rado no tiene en cuenta la información que se va obteniendo durante la campaña, por lo que proporciona el miso plan de producción para los tres escenarios de calidad. Como se muestra en la Figura, los planes sugieren producir en los mismos instantes de producción para $p \geq 0.3$, variando los productos debido al descenso de la calidad. Estrictamente hablando, los planes de producción proporcionados por la solución de valor esperado para D1 y D2 son no factibles, dado que las propiedades del fruto en el momento de producción no permiten producir los productos especificados.

Por otro lado, la solución al problema multietapa no depende de $p$. Proporciona distintos planes para cada escenario que siempre son factibles, dado que incorpora la información disponible en cada momento.

Finalmente, el Cuadro 2 incluye el valor de disponer de información perfecta (VPI) y el valor de la solución estocástica (VSS, i.e., la diferencia entre los beneficios obtenidos por la aplicación del problema multietapa frente al problema de valor medio) para los diferentes valores de $p$ considerados. Como se incluye en el Cuadro, el VSS es modesto, pero del mismo orden de magnitud que el VPI para todos los valores de $p$. Aún modesto, un incremento del $0.34 \%$ supone alrededor de $27,000 €$ por campaña para una molturación de 10 millones kg.

\section{Conclusiones}

Este trabajo ha planteado un método para obtener un plan de producción óptimo para la elaboración de AOV. Se han detallado los diferentes factores que se deben considerar y las relaciones entre variables. Finalmente se ha resuelto el problema propuesto para una serie de escenarios utilizando datos obtenidos a partir de expertos.

El método propuesto sirve como elemento fundamental para la construcción de un sistema de apoyo a la decisión para la producción de AOV, que junto con el sistema propuesto en [4], puede ayudar al operador en cada decisión del proceso productivo.

Las líneas de trabajo futuro incluyen la consideración de distintas variedades de fruto y la utilización de modelos estocásticos más sofisticados para la distribución de precios y demanda. 

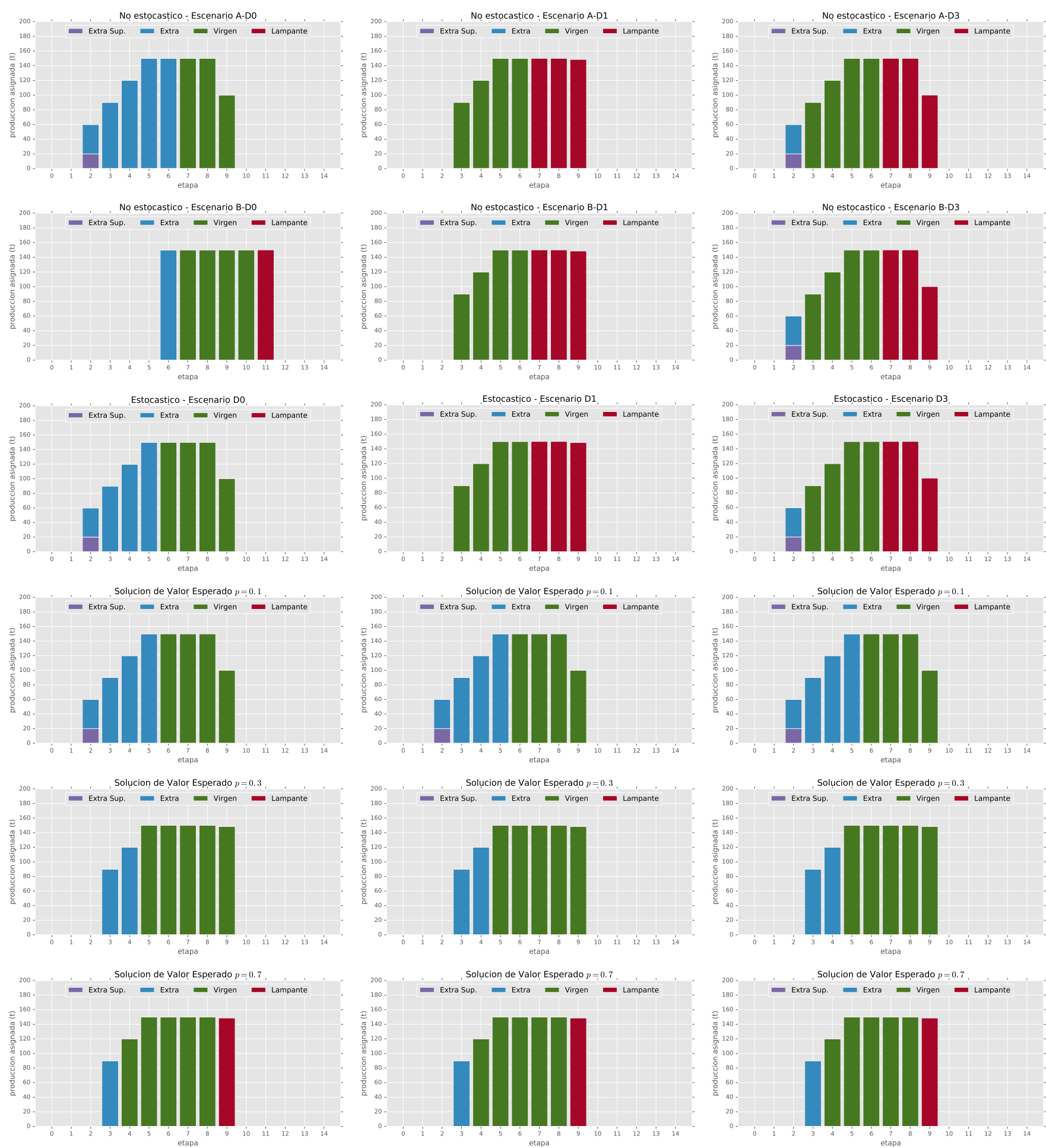

Figura 2: Planes de producción para los escenarios considerados en la Sección 3.2. Las columnas corresponden a las tres escenarios de calidad. Las columnas corresponden a los tres escenarios de evolución de la calidad. La primera y la segunda fila muestran el plan de producción óptimo suponiendo que existe información perfecta para los niveles de precios A y B respectivamente. La tercera fila presenta la solución al problema multietapa, mientras que las filas 4 a 6 muestran las solución del problema de valor esperado para $p=0.1, p=0.3$ y $p=0.7$, respectivamente. 


\section{Agradecimientos}

Este trabajo ha sido parcialmente subvencionado por los proyectos DPI2011-27284, TEP2009-5363 y AGR-6429. P. Cano Marchal ha sido beneficiario de una beca F.P.U. del Ministerio de Educación del Gobierno de España. Los autores agradecen a CM Europa los datos históricos de evolución de propiedades de aceitunas proporcionados.

\section{Referencias}

[1] Maria P. Aguilera, Gabriel Beltran, Sebastián Sanchez-Villasclaras, Marino Uceda, Antonio Jimenez, G. Beltrán, S.Sanchez-Villasclaras, Marino Uceda, A. Jiménez, Gabriel Beltran, Sebastián SanchezVillasclaras, Marino Uceda, and Antonio Jimenez. Kneading olive paste from unripe 'Picual' fruits: I. Effect on oil process yield. Journal of Food Engineering, 97(4):533-538, apr 2010.

[2] John R. Birge and Francois Louveaux. Introduction to Stochastic Programming. Springer, New York, edición: 2 edition, June 2011.

[3] P. Cano Marchal, J. Gómez Ortega, D. Aguilera Puerto, and J. Gámez García. Situación actual y perspectivas futuras del control del proceso de elaboración del aceite de oliva virgen. Revista Iberoamericana de Automática e Informática Industrial RIAI, 8(3):258-269, July 2011.

[4] P. Cano Marchal, J. Gámez García, and J. Gómez Ortega. Decision support system based on fuzzy cognitive maps and run-to-run control for global set-point determination. In 2015 IEEE International Conference on Systems, Man, and Cybernetics, 2015.

[5] P. Cano Marchal, D. Martínez Gila, J. Gámez García, and J. Gómez Ortega. Fuzzy decision support system for the determination of the set points of relevant variables in the virgin olive oil elaboration process. In 2013 IEEE International Conference on Systems, Man, and Cybernetics, pages 3489-3494, 2013.

[6] P. Cano Marchal, D. Martínez Gila, J. Gámez García, and J. Gómez Ortega. Optimal production planning for the virgin olive oil elaboration process. volume 19, pages 8921-8926, 2014.

[7] Maria Lisa Clodoveo. Malaxation: Influence on virgin olive oil quality. past, present and future - an overview. Trends in Food Science 83 Technology, 25(1):1323, May 2012.

[8] L. Di Giovacchino, N. Costantini, M. L. Ferrante, and A. Serraiocco. Influence of malaxation time of olive paste on oil extraction yields and chemical and organoleptic characteristics of virgin olive oil obtained by a centrifugal decanter at water saving. Grasas $Y$ Aceites, 53(2):179-186, 2002.

[9] L. Di Giovacchino, S. Sestili, and D. Di Vicenzo. Influence of olive processing on virgin olive oil quality. European Journal of Lipid Science and Technology, 104:587-601, 2002.

[10] Inc. Gurobi Optimization. Gurobi optimizer reference manual, 2015.

[11] F. Gutiérrez, B. Jiménez, A. Ruiz, and M. A. Albi. Effect of olive ripeness on the oxidative stability of virgin olive oil extracted from the varieties picual and hojiblanca and on the different components involved. Journal of Agricultural and Food Chemistry, 47(1):121-127, January 1999.

[12] William E. Hart, Carl Laird, Jean-Paul Watson, and David L. Woodruff. Pyomo - Optimization Modeling in Python, volume 67 of Springer Optimization and Its Applications. Springer US, Boston, MA, 2012.

[13] Antonio M. Inarejos-García, Aurora Gómez-Rico, M. Desamparados Salvador, and Giuseppe Fregapane. Influence of malaxation conditions on virgin olive oil yield, overall quality and composition. European Food Research and Technology, 228(4):671-677, February 2009 .

[14] B. Jiménez Herrera, A. Rivas Velasco, A. SánchezOrtíz, M. L. Lorenzo Tovar, M. Ubeda Munoz, R. M. Callejon, and E. Ortega Bernaldo de Ouiros. Influence of fruit maturation process on the sensory quality of virgin olive oils from picual, hojiblanca and picudo cultivars. Grasas Y Aceites, 63(4):403-410, December 2012. WOS:000311708500007.

[15] D.M. Martínez Gila, P. Cano Marchal, J. Gámez García, and J. Gómez Ortega. On-line system based on hyperspectral information to estimate acidity, moisture and peroxides in olive oil samples. Computers and Electronics in Agriculture, 116:1-7, aug 2015.

[16] Yves Pochet and Laurence A. Wolsey. Production Planning by Mixed Integer Programming. Springer Series in Operations Research and Financial Engineering. Springer New York, 2006.

[17] PoolRed. POOLred-Sistema de Información de Precios en Origen del Mercado de Contado del Aceite de Oliva, 2016.

[18] Jean-Paul Watson, David L. Woodruff, and William E. Hart. PySP: modeling and solving stochastic programs in Python. Mathematical Programming Computation, 4(2):109-149, June 2012. 